\title{
A review on experimental and clinical genetic associations studies on fear conditioning, extinction and cognitive-behavioral treatment
}

\author{
TB Lonsdorf and R Kalisch
}

Fear conditioning and extinction represent basic forms of associative learning with considerable clinical relevance and have been implicated in the pathogenesis of anxiety disorders. There is considerable inter-individual variation in the ability to acquire and extinguish conditioned fear reactions and the study of genetic variants has recently become a focus of research. In this review, we give an overview of the existing genetic association studies on human fear conditioning and extinction in healthy individuals and of related studies on cognitive-behavioral treatment (CBT) and exposure, as well as pathology development after trauma. Variation in the serotonin transporter ( $5 \mathrm{HTT}$ ) and the catechol-o-methyltransferase (COMT) genes has consistently been associated with effects in pre-clinical and clinical studies. Interesting new findings, which however require further replication, have been reported for genetic variation in the dopamine transporter (DAT1) and the pituitary adenylate cyclase 1 receptor (ADCYAP1R1) genes, whereas the current picture is inconsistent for variation in the brain-derived neurotrophic factor (BDNF) gene. We end with a discussion of the findings and their limitations, as well as future directions that we hope will aid the field to develop further. Translational Psychiatry (2011) 1, e41; doi:10.1038/tp.2011.36; published online 20 September 2011

\section{Introduction}

Learning to predict danger from previous experience is critical to an organism's survival. In fear conditioning, an environmental stimulus (conditioned stimulus, CS) comes to predict a naturally aversive stimulus (unconditioned stimulus, UCS) and thereby to induce a conditioned fear response (CR). ${ }^{1}$ After conditioning has occurred, the repeated presentation of the CS in the absence of UCS (exposure) leads to a gradual weakening of the $\mathrm{CR}$, a process referred to as extinction.

Fear conditioning and extinction represent basic forms of associative learning with considerable clinical relevance and have been implicated in the pathogenesis of anxiety disorders. ${ }^{2}$ Deficits in the extinction of learned fear associations have been observed in patients suffering from anxiety disorders like post-traumatic stress disorder (PTSD), phobias and panic disorder (PD). ${ }^{3,4}$ Further, extinction has inspired the clinical use of exposure to fear stimuli ${ }^{5}$ in cognitive-behavioral therapy (CBT), which is used to treat many forms of pathological anxiety. ${ }^{6,7}$ CBT represents a learning process leading to symptom relief and long-term changes in behavior that have measurable correlates in neural activation patterns, synaptic connectivity and gene expression patterns. ${ }^{8,9}$

Understanding the molecular pathways that mediate conditioning and extinction might therefore make an important contribution to the study of anxiety pathophysiology, resilience and treatment mechanisms, and open up new perspectives for pharmacological interventions. One promising, although by far not the only, strategy to identify molecular pathways in humans is genetic association studies.

Genetic association studies optimally investigate simple behavioral paradigms with sufficient inter-individual variability and clear heritability that elicit robust behavioral responses, which are easy to measure and quantify and rely on a welldefined underlying neural circuitry. Fear conditioning and extinction fulfill these criteria.

First, both human ${ }^{10,11}$ and animal studies ${ }^{12}$ show that there is considerable inter-individual variability in the ability to acquire and extinguish conditioned fear as well as in profiting from CBT, and that genetic factors represent a significant source of this variation. Specifically, one-third of the variance in human fear conditioning ${ }^{10}$ and in the vulnerability for anxiety disorders ${ }^{13}$ is attributed to genetic factors.

Second, conditioned fear can be easily and reliably measured using, for example, skin conductance responses (SCRs) and/or fear potentiated startle (FPS) responses (see Table 1 for explanation of technical terms). Importantly, twin studies have proven the reliability of both SCRs ${ }^{10}$ and FPS ${ }^{11}$ for heritability studies.

Third, the neural network underlying fear conditioning and extinction has been studied intensively in both animals ${ }^{14,15}$ and humans. ${ }^{16} \mathrm{~A}$ well-delineated neural network is not only advantageous for genetic imaging studies, but may also guide selection of candidate genes.

Institute for Systems Neuroscience, University Medical Center Hamburg-Eppendorf (UKE), Hamburg, Germany

Correspondence: Dr TB Lonsdorf, Institute for Systems Neuroscience, University Medical Center Hamburg-Eppendorf (UKE), Martinistrasse 52, Hamburg 20246, Germany.

E-mail: t.lonsdorf@uke.uni-hamburg.de

Keywords: anxiety; cognitive-behavioral treatment; conditioning; extinction; exposure; genetics

Received 16 May 2011; revised 29 June 2011; accepted 30 July 2011 
Table 1 Explanation of technical terms and abbreviations

\begin{tabular}{|c|c|}
\hline Term & Explanation \\
\hline $\begin{array}{l}\text { Fear potentiated startle } \\
\text { (FPS) }\end{array}$ & $\begin{array}{l}\text { Augmentation of the startle reflex by a } \\
\text { fearful state, for example, induced by } \\
\text { a certain stimulus }\end{array}$ \\
\hline Dark-enhanced startle & $\begin{array}{l}\text { Augmentation of the startle reflex by } \\
\text { darkness }\end{array}$ \\
\hline $\begin{array}{l}\text { Skin conductance response } \\
\text { (SCR) }\end{array}$ & $\begin{array}{l}\text { The alteration in the electrical } \\
\text { resistance of the skin associated with } \\
\text { psychological or physiological arousal }\end{array}$ \\
\hline $\begin{array}{l}\text { Unconditioned stimulus } \\
\text { (UCS) }\end{array}$ & $\begin{array}{l}\text { In experimental human studies often } \\
\text { an aversive electrotactile stimulation } \\
\text { or an air puff to the eye }\end{array}$ \\
\hline CS+ & Stimulus that predicts the UCS \\
\hline $\mathrm{CS}-$ & $\begin{array}{l}\text { Stimulus that does not predict the } \\
\text { UCS }\end{array}$ \\
\hline CS+ potentiation & $\begin{array}{l}\text { Augmentation of a reaction (e.g., FPS) } \\
\text { elicited by/during the CS+ as } \\
\text { compared to a reaction elicited by/ } \\
\text { during the ITI }\end{array}$ \\
\hline CS- potentiation & $\begin{array}{l}\text { Augmentation of a reaction (e.g., FPS) } \\
\text { elicited by/during the CS- as } \\
\text { compared to a reaction elicited by/ } \\
\text { during the ITI }\end{array}$ \\
\hline $\mathrm{CS}+/ \mathrm{CS}-$ discrimination & $\begin{array}{l}\text { Augmentation of a reaction (e.g., FPS) } \\
\text { elicited by/during the CS+ as } \\
\text { compared to a reaction elicited by/ } \\
\text { during the CS- }\end{array}$ \\
\hline Inter-trial interval (ITI) & $\begin{array}{l}\text { Time between two stimulus } \\
\text { presentations; here: time between } \\
\text { two CS's }\end{array}$ \\
\hline
\end{tabular}

In this review, we summarize existing findings, sorted by molecular pathways, covering conditioning and extinction in healthy individuals, CBT and exposure outcome in clinical populations, as well as PTSD development after trauma. We try to propose mechanistic interpretations, critically discuss limitations and pitfalls, and show up interesting new directions for future research.

\section{Serotonin}

Although the serotonin (5-HT) system presents with a multitude of promising candidate genes, only polymorphisms in the serotonin transporter (5-HTT) gene, which is responsible for presynaptic 5-HT reuptake (for a review, see ref. 17), and the monoamine oxidase $\mathrm{A}(M A O-A)$ gene, which degrades $5-\mathrm{HT}$ (for a review, see ref. 18), have been studied with respect to fear conditioning and extinction processes.

5-HTTLPR. 5-HTT presents with a $43 \mathrm{bp}$ insertion/deletion polymorphism in its promoter region, which is referred to as 5-HTT linked polymorphic region (5-HTTLPR) and most commonly comprises a short (s) and a long (I) variant. The s-allele is associated with $\sim 50 \%$ reduced transcriptional activity in vitro, ${ }^{19}$ but human in vivo or post-mortem studies failed to reveal consistent functional effects, ${ }^{20-22}$ probably because the polymorphism exerts its effect during early neurodevelopment (for example, ref. 23).
The G-allele of a functional $A / G$ single-nucleotide polymorphism (SNP, rs25531) upstream of the 5-HTTLPR ${ }^{24}$ is almost always in phase with the 5-HTTLPR I-allele ${ }^{25}$ and is associated with reduced 5-HTT transcriptional efficacy. ${ }^{24,26}$ 5-HTTLPR and rs25531 are often combined as a functional mini-haplotype ('tri-allelic 5-HTTLPR'). The I-allele of the 5-HTTLPR is thereby further subdivided into $L_{A}$ and $L_{G}$. Functionally, the $L_{G}$-allele is equivalent to the low expressing 5-HTTLPR s-allele, ${ }^{26}$ and grouping of individuals based on the triallelic 5-HTTLPR is based on inferred 5-HTT expression levels. $^{26}$

Three experimental and five clinical studies have to date investigated an association of the bi- and/or triallelic 5-HTTLPR with fear conditioning- and/or extinction-related processes.

Garpenstrand and co-workers ${ }^{27}$ selected 20 good and 20 bad performers from a cohort of 346 fear-conditioned subjects, on the basis of their SCR discrimination, during conditioning, between a CS paired with the UCS $(\mathrm{CS}+)$, and a control stimulus never paired with the UCS (CS-) (see Tables 2 and 3 for details on design and sample). Testing for $\mathrm{CS}+/ \mathrm{CS}-$ discrimination is an appropriate means to control for general sensitization and stimulus responsivity effects. The authors observed an over-representation of the 5-HTTLPR s-allele in the good performers and, accordingly, significantly more SCR discrimination $\left(\mathrm{CS}+>\mathrm{CS}_{-}\right)$) in s-allele carriers than in non-carriers. This effect was maintained during (immediate) extinction on a descriptive level $(P=0.11)$.

Lonsdorf and co-workers ${ }^{28}$ replicated and extended the above findings in a sample of 48 volunteers, partly selected a priori for their 5-HTTLPR and COMTval158met (see below) genotypes. Eyeblink startle responses were induced by presenting auditory (startle probe) probes during both types of CSs and during the inter-trial interval (ITI, see Table 1 for explanations of technical terms). S-carriers displayed significantly more FPS CS + potentiation $(\mathrm{CS}+>\mathrm{ITI})$ during acquisition than non-carriers, in the absence of significant differences in $\mathrm{CS}+/ \mathrm{CS}$ - discrimination, CS- potentiation (CS->ITI) or ITI raw startle (untransformed ITI scores elicited during the ITI). In addition, while s-carriers showed the expected conditioning-related effects (significant CS+ and $\mathrm{CS}-$ potentiation, $\mathrm{CS}+/ \mathrm{CS}-$ discrimination), these effects were absent in non-carriers. During the $24 \mathrm{~h}$ delayed extinction phase, s-carriers again showed significantly more $\mathrm{CS}+$ potentiation, but also less CS - inhibition (CS $-<\mathrm{ITI}$, an effect that is taken to reflect the learned safety of the CS-), in the absence of group differences in $\mathrm{CS}+/ \mathrm{CS}$ - discrimination or ITI raw startle. However, using SCR, no learning-related group differences were observed, whether during conditioning or extinction (see below for a discussion of the different measurements).

Finally, Crisan and co-workers ${ }^{29}$ reported an association between the 5-HTTLPR s-allele and enhanced observational fear learning ${ }^{30}$ in 32 participants. In this paradigm, s-carriers displayed marginally higher SCRs when observing a model (that is, another person) being presented with the CS + or the UCS, but not when the model was presented with the CS-. During subsequent testing, s-carriers displayed significantly higher SCRs to $\mathrm{CS}+\mathrm{s}$, but not to $\mathrm{CS}-\mathrm{s}$, presented to 


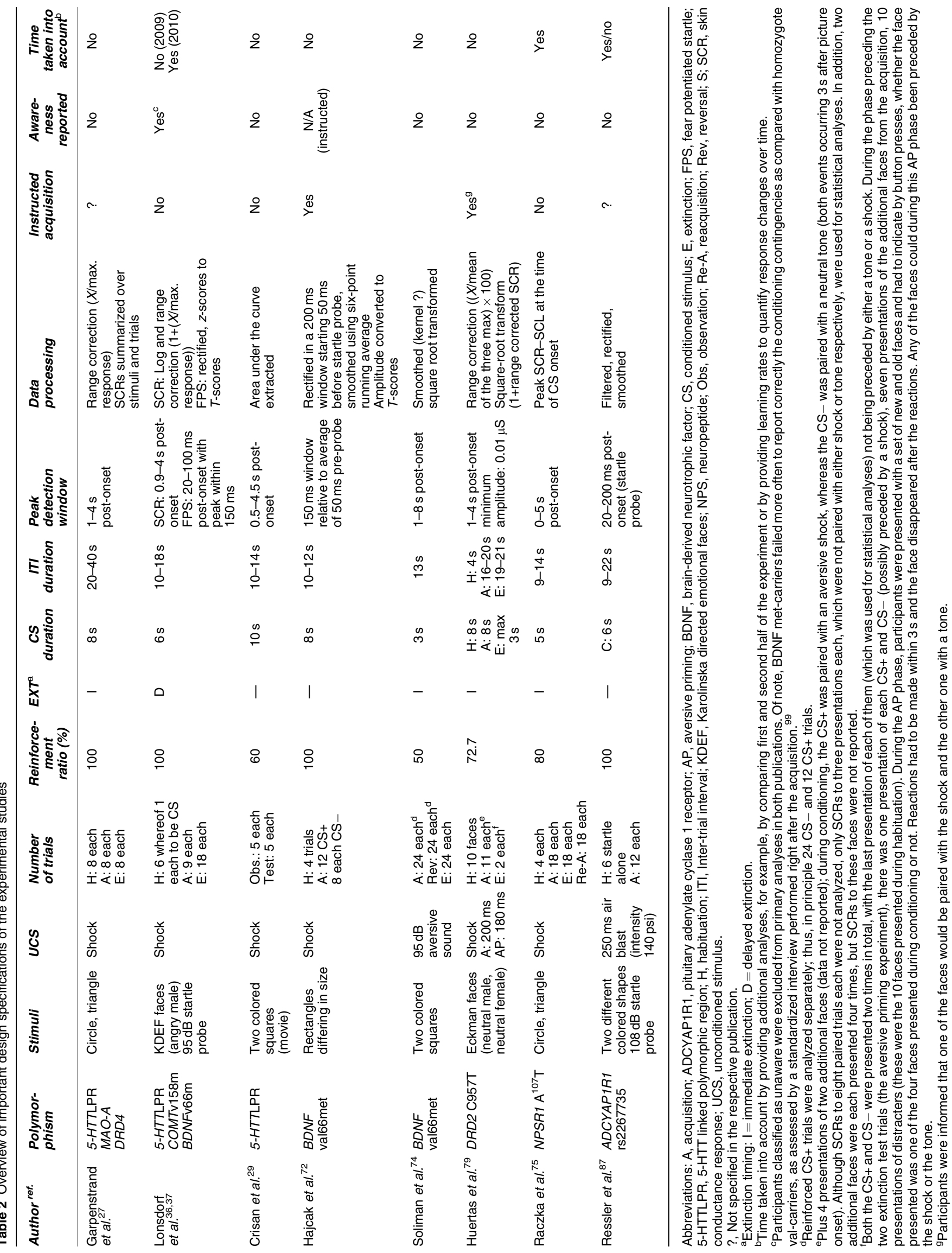




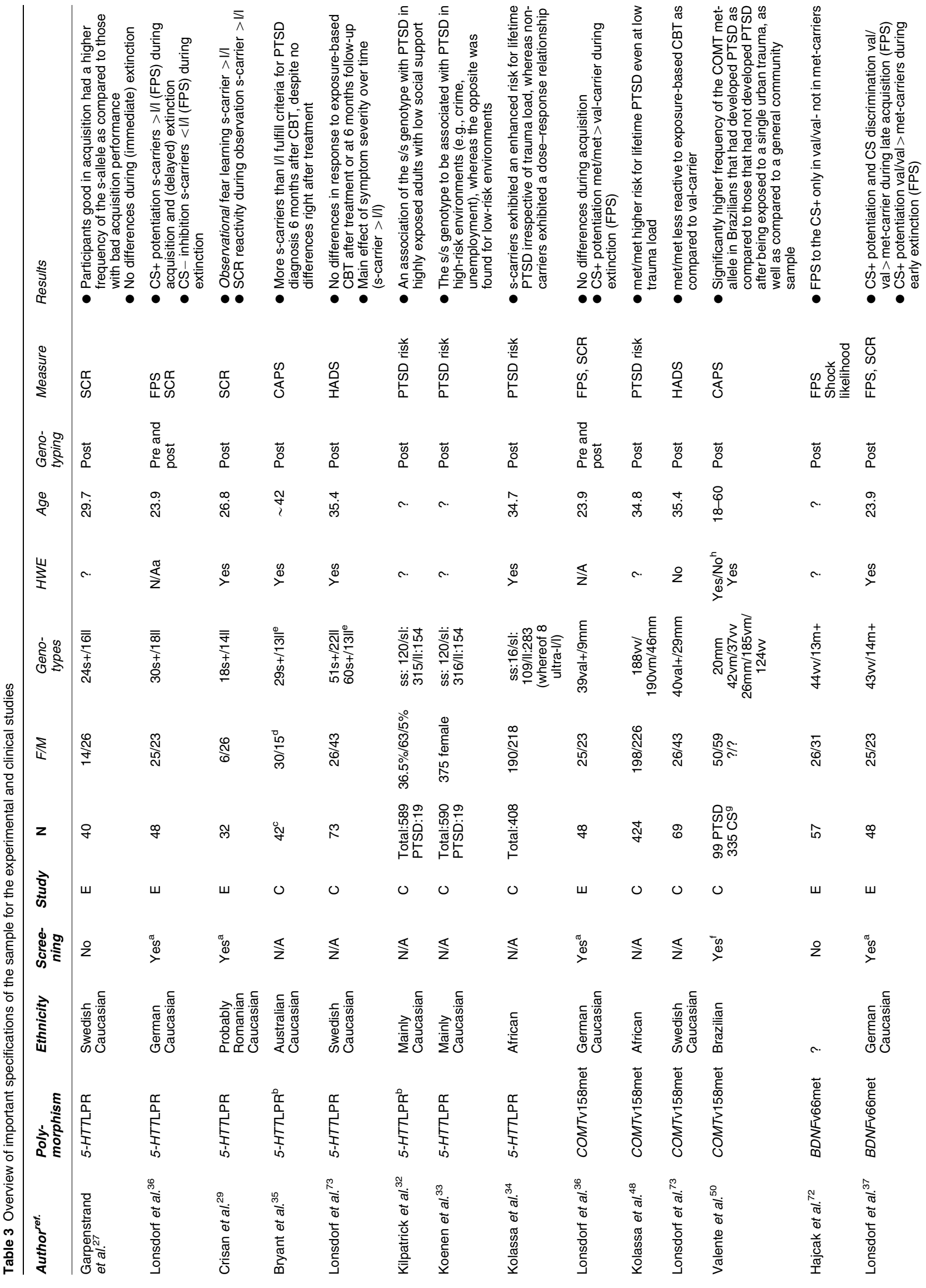




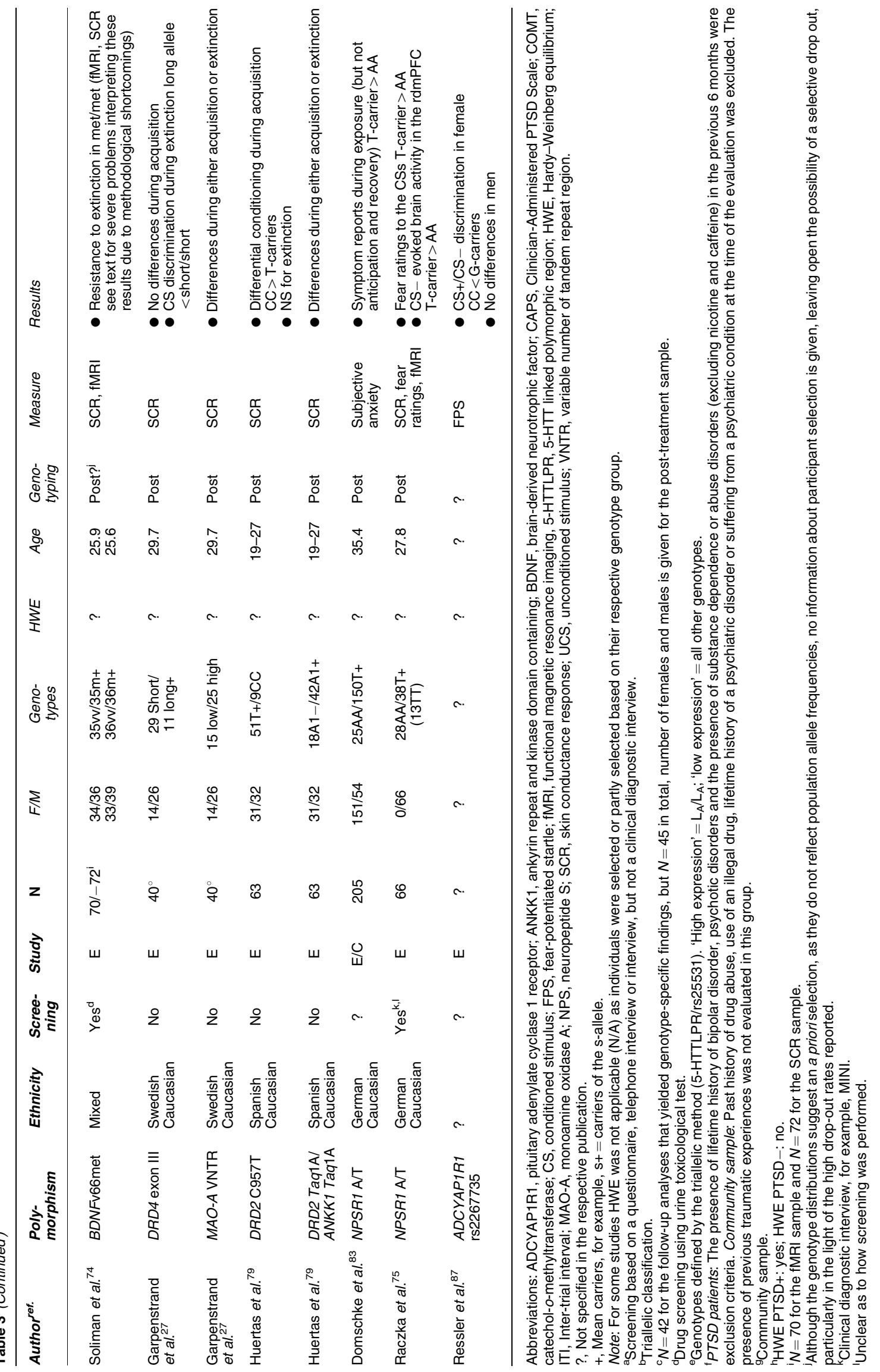


themselves in the absence of the UCS. Group differences were reported in analyses that tested SCRs to the CS + and the CS- separately; however, no statistics on CS +/CSdiscrimination was given.

In sum, three experimental studies reported facilitated fear learning in 5-HTTLPR s-allele carriers in at least one psychophysiological modality (SCR or FPS), an effect that appears to carry over into subsequent extinction. Importantly, as far as reported, groups did not differ in the intensity levels chosen for UCS presentations, ${ }^{27,28}$ in SCRs to received UCSs, or in ITI raw startle (ref. 28).

PTSD is the prototypical anxiety disorder where fear conditioning makes an unquestionable contribution to disease aetiology (for example, see ref. 31). If 5-HTTLPR genotype affects fear conditioning propensity, it should also be associated with PTSD vulnerability. Three epidemiological studies support this claim and thus underscore the translational potential of conditioning genetics.

In a sample of hurricane victims $(N=589)$, PTSD risk was enhanced in individuals carrying the s/s genotype if they also received low social support ${ }^{32}$ or if they also lived in high-risk environments (characterized, for example, by crime or unemployment). ${ }^{33}$ By contrast, s/s-carriers had a lower risk to develop PTSD in low-risk environments. ${ }^{33}$ However, both analyses were limited by the very low number of individuals with a current PTSD diagnosis $(N=19$, whereof $n=4$ 5-HTTLPR s/s-carriers). Finally in a study in 424 unrelated refugees of the Rwandan civil war, Kolassa and co-workers found an enhanced risk for lifetime PTSD in s/s-carriers irrespective of trauma load (as assessed 12-13 years later by counting the number of different traumatic event types experienced/witnessed), whereas I-carriers (s/l and I/I) exhibited the expected dose-response relationship between trauma load and lifetime risk. ${ }^{34}$ At very high traumatic load however ( $>15$ events), no differences in lifetime risk were found between the genotype groups, suggesting that the influence of genetics decreases with increasing trauma load.

Hence, the clinical data are in agreement with the idea that low 5-HTT expression is associated with facilitated and more persistent fear conditioning, whereas high 5-HTT expression is associated with abnormal resistance to fear conditioning. However, it remains elusive if the apparent persistence of fear simply reflects a carryover of stronger fear into later exposure or perhaps deficits in the corrective safety learning that characterizes extinction. Unfortunately, the preclinical studies did not assess rates of extinction as one means to quantify learning. However, provided one accepts the idea of extinction learning as the major active ingredient to CBT, two recent therapy studies permit interesting conclusions.

Bryant and co-workers ${ }^{35}$ investigated 42 unmedicated PTSD patients who were provided with weekly 90-min individual CBT sessions for 8 weeks. CBT reduced symptoms equally in both groups, and there were no significant genotype group differences in symptom scores before and immediately after treatment, significantly more individuals with inferred low 5-HTT expression (s- and $\mathrm{L}_{\mathrm{G}_{-}}$-carriers) met PTSD diagnosis 6 months after treatment and also reported more symptoms

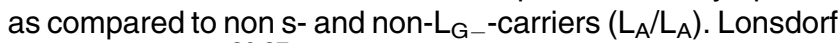
and co-workers ${ }^{36,37}$ reported a similar finding of persistently higher symptom scores in s- and $\mathrm{L}_{\mathrm{G}}$-carriers in $69 \mathrm{PD}$ patients treated with weekly CBT sessions (regular group or internet-based CBT) for 10 weeks. In contrast to the study by Bryant and co-workers ${ }^{35}$ group differences in symptom scores reached significance also at pre- and post-treatment. Because in both studies, acute symptom reduction thought CBT succeeded equally well in both genotype groups (excluding deficits in corrective safety learning in s- and $L_{G}$-carriers), the explanation of the group differences of the 6-month follow-up scores most likely be sought in the persistence and durability of the fear memories generated during trauma.

It should be noted that there are currently no twin studies showing heritability of CBT. Nevertheless, if taken together, existing data on the bi- and triallelic 5-HTTLPR genotype yield an impressively consistent picture across preclinical-experimental, epidemiological and therapy studies, making 5-HTTLPR a prime example for successful translation of biochemical and molecular-genetic findings into human pathophysiological research.

MAO-A VNTR. The human MAO-A gene contains an untranslated variable number of tandem repeat region $(M A O-A \text { uVNTR })^{38}$ that yields six different alleles that vary in transcriptional efficiency $(2 \mathrm{R}<3 \mathrm{R}<3.5 \mathrm{R}=4 \mathrm{R})$. Functional data are inconsistent for the $5 R^{38,39}$ and absent for the $6 \mathrm{R}$-allele (for a review, see ref. 23).

Garpenstand and co-workers ${ }^{27}$ found no differences in SCR conditioning and extinction between individuals with putatively high $(3.5 \mathrm{R} / 4 \mathrm{R})$ or low $(3 \mathrm{R} / 5 \mathrm{R}) M A O-A$ expression levels in an additional analysis of the sample described above.

\section{Dopamine}

Like 5-HT, the dopamine (DA) system yields a multitude of promising candidate genes, and studies on fear conditioning, extinction, CBT and PTSD development after trauma have investigated associations with polymorphisms in the catechol-O-methyltransferase (COMT), DA transporter (DAT1), D2 (DRD2) and D4 (DRD4) receptor genes.

COMT val158met (rs4680). COMT degrades extracellular DA (for a review, see ref. 40) and is of primary importance in the prefrontal cortex, but less so in striatal areas. ${ }^{41}$ The COMT gene harbors a functional A/G SNP, leading to the substitution of the amino-acid valine by methionine at codon 158 (COMTval158met). Homozygosity for the met allele leads to four times reduced enzymatic activity compared to homozygosity for the val-allele, ${ }^{42}$ and thereby affects effectiveness of DA degradation by COMT and the availability of synaptic DA (higher in met-carriers). ${ }^{43}$

Two experimental and three clinical studies have to date investigated an association of COMTval158met with fear conditioning and/or extinction processes.

In a sample of 48 volunteers, partly selected a priori for COMTval158met genotype (and 5-HTTLPR, see above ${ }^{28}$ ) Lonsdorf and co-workers ${ }^{37}$ reported no association of COMTval158met genotype with FPS and SCR conditioning. However, during 24-h delayed extinction, met/met-carriers showed significantly enhanced CS + potentiation compared 
to val-carriers, suggesting resistance to extinction. No group differences in CS + /CS - discrimination, CS - potentiation, raw ITI startle or SCRs were observed. As a limitation of this study, the low number of homozygous met-carriers has to be mentioned.

In a subsequent clinical study, the same group ${ }^{37}$ also investigated the efficacy of exposure-based CBT in 69 PD patients (see also above). Supporting the notion of extinction resistance, met/met-carriers seemed to benefit less from exposure-based treatment modules (vs cognitive modules) than val-carriers. Hence, COMT met/met-carriers do not seem to differ from val-carriers in their conditionability, but in their ability to use corrective experience for fear reduction, which is in line with the met-allele being associated with emotional perseveration, reduced cognitive flexibility, but enhanced stability. ${ }^{44,45}$

Raczka and co-workers ${ }^{46}$ investigated 69 healthy male participants selected a priori based on their COMTval158met genotype (and a DAT1 VNTR, see below) in an experiment involving conditioning, immediate extinction and immediate reconditioning. Like in the first COMT study, ${ }^{26}$ COMT genotype had no measurable effect on indices of conditioning (SCR as well as subjective fear ratings intermittently provided throughout the experiment). However, there was also no association with extinction learning in SCR and fear ratings, as well as in a computational analysis of fear rating time series by virtue of a formal reinforcement learning model. The latter provides a possibility to estimate extinction learning rates and thus to gain a more fine-grained picture of the associative processes occurring during an exposure phase than simple averaging of CR scores. An apparent methodological difference to previous work was the use of immediate extinction, excluding potential effects of long-term fear memory consolidation processes on CRs measured in extinction. It is therefore possible that the extinction resistance observed by Lonsdorf and co-workers ${ }^{28}$ reflects better fear memory consolidation in met/met-carriers rather than a deficit in safety learning. In this context, it is worth noting that DA has been implicated in memory consolidation processes in animal studies (for example, ref. 47).

Like the enhanced and persistent fear conditioning in low 5-HTT-expressing individuals, the putatively enhanced fear memory consolidation in COMT met/met-carriers should be associated with enhanced risk for PTSD. Two epidemiological studies support this prediction. Kolassa and co-workers ${ }^{48}$ observed that met/met-carriers, after experiencing at least one traumatic event, had a high risk for lifetime PTSD. By contrast, val-allele carriers showed the typical dose-dependent increase of lifetime PTSD risk with increasing trauma load. In analogy to the pattern observed with respect to 5-HTTLPR genotype, the 'risk' genotype (met/met) conferred a higher lifetime PTSD risk in particular at lower trauma loads, and differences between the genotype groups vanished at high traumatic load ( $>15$ events), again suggesting that the influence of genetics decreases with increasing trauma load. Importantly, genotype groups did not differ in the number or types of traumatic events experienced, rendering a gene-environment correlation (for example, exposure to trauma may depend on the individual's genotype), ${ }^{49}$ rather unlikely.
In a similar vein, Valente and co-workers ${ }^{50}$ found a significantly higher frequency of the COMTval158met metallele in Brazilians who had developed PTSD after a single urban trauma than in individuals resilient to PTSD and in a community sample. Further, trauma-exposed individuals carrying a met-allele reported significantly more PTSD symptom severity than non-carriers. Limitations of this study include the rather small sample sizes for trauma exposed individuals $(N=99$, whereof 34 resistant to PTSD) and different genotype and allele frequencies in the three groups. As trauma exposure was not assessed in the community sample and different allele frequencies were observed in the different groups, a gene-environment correlation cannot be finally excluded.

In sum, the current literature points toward an important role for COMTval158met in fear memory consolidation, which also affects extinction success once sufficient time for consolidation of the fear memory has elapsed. Because exposure therapy occurs with a considerable delay to trauma, COMTval158met genotype might turn out as a predictor of treatment response.

DAT1 VNTR (rs28363170). The DAT mediates DA reuptake and thus regulates the duration and amplitude of DAergic signaling, particularly in striatal areas. ${ }^{51}$ The DAT1 gene harbors a $40 \mathrm{bp}$-VNTR polymorphism in its $3^{\prime}$-untranslated region that most frequently occurs as 9 or 10 tandem repeats $(R)$.

Of those studies finding VNTR effects on DAT expression, cell-based assays majorily indicate that the 9R-allele reduces expression, ${ }^{52-54}$ whereas evidence from human studies is split. ${ }^{55-58}$ According to current models, reduced DAT expression should amplify phasic DA signals. ${ }^{51}$

In their above sample, Raczka and co-workers ${ }^{46}$ used formal computational modeling (see above) to show higher learning rates during extinction (but not conditioning) in DAT1 9R-carriers as compared to non-carriers. Of note, standard analyses comparing phase-averaged SCR and rating scores showed no group differences. Higher learning rates were accompanied by higher activation of the ventral striatum to the unexpected UCS omission in extinction. In associative learning theory, such 'prediction errors' are supposed to drive association formation (here, between the CS and the absence of the UCS) and phasic ventral-striatal DA release is currently the prime candidate for prediction error encoding in appetitive conditioning. ${ }^{59}$ Drawing an analogy between learning to expect safety (in extinction) and learning to expect reward (in appetitive conditioning), the authors suggested a contribution of the meso-striatal DA system to extinction learning. No group differences in striatal prediction error encoding were observed in the conditioning phase.

DRD2 C957T (rs6277). The synonymous SNP in the DRD2 gene, DRD2 C957T (rs6277), was initially assumed to be functionally silent. Later, the T-allele was associated with decreased mRNA stability and protein synthesis in vitro ${ }^{60}$ and higher DRD2 receptor affinity $(\mathrm{C} / \mathrm{C}<\mathrm{C} / \mathrm{T}<\mathrm{T} / \mathrm{T}) .{ }^{61}$

In a sample of 60 individuals, Huertas and co-workers ${ }^{62}$ found T-carriers to display significantly lower SCRs to CS $+\mathrm{s}$ in one late compared with one early conditioning trial (see 
Table 2 for details). Non-carriers $(\mathrm{C} / \mathrm{C})$ in turn tended to show an increase. No differences between the genotype groups were found in CS- and UCS SCRs. A formal test of SCR discrimination (CS $+>\mathrm{CS}-$ ) was not reported. In extinction, no differences between the genotype groups were found. Limitations of the study include very unequal sample sizes and the use of single trials $(N=1-3)$ for statistical analyses (see Table 3).

DRD4R VNTR. The DRD4 gene contains a VNTR polymorphism of a $48 \mathrm{bp}$ sequence that affects $\mathrm{D} 4$ receptor function in vivo. ${ }^{63}$ The $7 \mathrm{R}$ variant leads to decreased inhibitory post-synaptic DA effects compared with the $4 \mathrm{R}$ and the $2 \mathrm{R}$ forms. ${ }^{64}$ Caucasians are mostly grouped as $7 \mathrm{R}$ carriers vs non-carriers, but a new suggestion for functional classification has been proposed recently. ${ }^{65,66}$

Garpenstand and co-workers ${ }^{27}$ (see above) found no DRD4R VNTR genotype variant (long: $6-8 \mathrm{R}$ vs short: $2-5 \mathrm{R}$ ) over-represented in good or poor conditioning performers. Although no difference in SCRs were found during conditioning, long-allele carriers showed significantly more CS + /CSdiscrimination during extinction. However, this association did not survive correction for multiple comparisons. In addition, extinction results in this sample must be interpreted in the awareness that participants were selected based on extreme performance during conditioning (see above).

\section{Brain-derived neurotrophic factor}

BDNF val66met. Brain-derived neurotrophic factor (BDNF) is the most abundant neutrophin in the central nervous system and is implicated in synaptic plasticity. ${ }^{67}$ The human $B D N F$ gene harbors a functional G/A SNP in its pro-domain, leading to a valine to methionine substitution in codon 66 (BDNFval66met). The met-allele is associated with impairments in intracellular trafficking and activity-dependent BDNF secretion. ${ }^{68,69}$

Animal work has implicated BDNF in hippocampus ${ }^{70}$ and amydala-dependent ${ }^{71}$ learning and memory, and to date, three human studies exist.

Hajcak and co-workers ${ }^{72}$ used a fear generalization paradigm in 57 participants. A rectangle served as CS +, and three different rectangles, differing gradually in size from the $\mathrm{CS}+$, served as CS-s (see Table 2 for details). A significant stimulus $\times$ genotype interaction on FPS was observed in the absence of differences in ITI startle reactivity, chosen UCS (shock) intensity or UCS likelihood ratings. Homozygous val-carriers showed significantly higher FPS to the $\mathrm{CS}+$ than met-carriers, relative to the CS- that was maximally dissimilar from the $\mathrm{CS}+$. No differences in FPS to the various $\mathrm{CS}-\mathrm{s}$ were observed.

Similarly, Lonsdorf and co-workers ${ }^{73}$ reported in a sample of 48 individuals more pronounced FPS CS + potentiation and $C S+/ C S$ - discrimination in val-carriers as compared to non-carriers during late (but not early) conditioning. This carried over to the early (but not late) extinction phase $24 \mathrm{~h}$ later, manifesting as significantly more pronounced CS+ potentiation in homozygous val-carriers. Because genotype groups had reached similar fear reduction at the end of extinction, this most likely reflects enhanced fear memory retrieval, rather than a safety learning deficit. No difference was found in SCR discrimination. Both studies were limited by unequal numbers in the two genotype groups (see Table 3).

Soliman and co-workers recently ${ }^{74}$ published a paradigm consisting of a conditioning, a reversal learning and an extinction phase following immediately upon each other in a sample that consisted of an equal number of met-carriers and non-carriers (total $N=72$ ). During reversal learning, the stimulus that had served as CS + during conditioning now served as the CS- and vice versa, and in extinction, both stimuli were unpaired (see Table 2 for details).

During fear conditioning, met-carriers showed an overall heightened SCR to both $\mathrm{CS}+$ and $\mathrm{CS}-$ in the absence of group differences in SCR discrimination (CS $+>\mathrm{CS}-$ ). Stronger CS- responses during late conditioning in metcarriers than in val-homozygotes were interpreted as a deficit in safety learning. No SCR data from the subsequent reversal phase were reported. During extinction, there were again generally heightened SCRs in met-carriers. Specifically, during late extinction, responses to the $\mathrm{CS}+(=\mathrm{CS}-$ in reversal) were higher in met-carriers. $\mathrm{CS}+/ \mathrm{CS}-$ discrimination and $\mathrm{CS}-(=\mathrm{CS}+$ in reversal) responses were not reported. This and the unorthodox reversal manipulation preceding the extinction phase (resulting in the CS + already being consistently presented unpaired with the UCS before extinction) calls for further qualification of the authors' interpretation of the data as reflecting an extinction deficit in met-carriers. A concurrent finding of decreased brain activation during extinction in met-carriers in the ventromedial prefrontal cortex and enhanced activation in the amygdala to $\mathrm{CS}+\mathrm{S}(=\mathrm{CS}-$ in reversal) relative to a fixation baseline would also require further information about preceding activations in conditioning and reversal, as well as responses to $\mathrm{CS}-(=\mathrm{CS}+$ in reversal) and $\mathrm{CS}+$ vs $\mathrm{CS}-$ contrasts to draw firm conclusions. So far, it cannot be excluded that the results merely reflect the generally heightened CS responsivity in met-carriers.

The picture that emanates from these three studies is relatively inconsistent, the strongest overlap lying in the enhanced FPS conditioning in val-homozygotes. In an attempt to shed further light on potential BDNF genotype effects, we reanalyzed SCR and fear rating data from a previously published data set using a continuous conditioningextinction-reconditioning paradigm in 46 val-homozygotes vs 23 met-carriers $^{75}$ (see Supplementary Information). Homoyzgous val-carriers showed generally heightened SCRs to both $\mathrm{CS}+\mathrm{s}$ and $\mathrm{CS}-\mathrm{s}$ during reconditioning only, in the absence of any group differences in discrimination. In fear ratings, val-homozygotes showed less CS + /CS - discrimination, caused by lower fear ratings to $\mathrm{CS}+\mathrm{s}$, relative to met-carriers, in both conditioning and reconditioning. Learning rates showed no genotype effects. Hence, these data rather enhance the disagreements currently existing in the literature.

To sum up, no clear picture emerges currently from data on the BDNFval66met genotype (for differences in design and methods see Tables 1 and 2) and results must be treated preliminary until replicated by independent laboratories. As animal studies have implicated BDNF in hippocampusdependent learning and human studies have shown 
associations of this SNP with hippocampus-dependent processes, ${ }^{69}$ context conditioning, relying heavily on the hippocampus, may be a more promising candidate for future studies.

\section{Other systems}

ANKK1 Taq1A (rs1800497). The novel ankyrin repeat and kinase domain containing (ANKK1) gene is involved in signal-transduction pathways ${ }^{76}$ and harbors the Taq1A restriction fragment length polymorphism (Glu713Lys). The polymorphism was initially thought to be located within the nearby $D R D 2$ gene, but from the current state of knowledge, its initial association with altered D2 receptor density ${ }^{77,78}$ is problematic.

Huertas and co-workers ${ }^{79}$ (see above) found no association of the $A N K K 1$ Taq1A restriction fragment length polymorphism with fear learning and (immediate) extinction. As for the authors' analysis of DRD2 C957T in the same data set, unequal group sizes and the use of single trials for statistics (see above and Table 3 ) have to be mentioned as a limitation.

NPSR1 Asn ${ }^{107}$ Ile (rs324981). Neuropeptide S (NPS) is a recently discovered neuropeptide that animal studies have implicated in arousal, anxiety and fear learning (for a review, see ref. 80). The human NPS receptor gene NPSR1 harbors a functional $A / T$ SNP, leading to an amino-acid exchange from aspargine to isoleucine $\left(A s n^{107} \| l e\right)$. The T-allele is associated with increased NPSR cell surface expression and 10-fold enhanced efficacy of NPS at NPSR in vitro. ${ }^{81,82}$

Raczka and co-workers ${ }^{75}$ (see above) performed conditioning, immediate extinction and immediate reconditioning in 66 healthy male volunteers. SCR results during the three phases revealed no genotype group differences in CS + /CSdiscrimination or general CS responsivity. By contrast, $\mathrm{T}$-allele carriers gave higher $\mathrm{CR}$ ratings to both $\mathrm{CS}+\mathrm{s}$ and $\mathrm{CS}-\mathrm{s}$ during conditioning (reappearing at trend level in reconditioning), suggesting that they may consciously overperceive or over-interpret their conditioned responses. This was accompanied by CS + hyper-responsivity of an area in the dorsal-medial prefrontal cortex previously associated with conscious threat appraisal. ${ }^{16}$

Paralleling these results, Domschke and co-workers ${ }^{83}$ showed in 205 PD patients with agoraphobia that T-allele carriers report significantly stronger increases in perceived symptom intensity elicited by a panic-relevant stimulus (sitting in a small locked dark chamber) again in the absence of a corresponding genotype effect on physiological responding (heart rate).

Hence, there is converging evidence from two studies that the T-allele of the NPSR1 Asn ${ }^{107}$ Ile SNP may be associated with amplified subjective experience and interpretation of fear reactions or stimuli, in the sense of catastrophizing over-interpretations, which is thought to be crucial for the development and maintenance of PD. ${ }^{84,85}$ However, whether this SNP is also associated with disease-relevant fear learning and/or extinction processes remains an open question.
ADCYAP1R1 C/C (rs2267735). The pituitary adenylate cyclase-activating protein (PACAP) stimulates cAMP production in the anterior pituitary ${ }^{86}$ and exerts pleiotropic functions in development, metabolism and cell signaling (cf. ref. 87)

Ressler and co-workers ${ }^{87}$ identified the $\mathrm{C} / \mathrm{C}$ genotype of an SNP in the ADCYAP1R1 gene to be associated with PTSD in female, but not male, highly traumatized urban civilian subjects using a tag-SNP approach. In a sample of PTSD patients (see Table 3 ), they also observed an association between the C/C genotype and impaired CS + /CS - startle discrimination during late conditioning, again restricted to females. Separate analyses for CS,$+ \mathrm{CS}_{-}$and ITI startle responses were not reported, and thus it remains unclear as to whether the effect was due to impaired excitatory (less CS + responding) or inhibitory (too much $\mathrm{CS}$ - responding) learning. In support of amplified excitatory responding, females with the $\mathrm{C} / \mathrm{C}$ genotype also showed significantly increased dark-enhanced startle than non-carrier females, whereas again no differences were found in males.

In sum, there is new promising evidence for a possible association of an ADCYAP1R1 SNP with fear learning.

\section{Summary}

In our summary of genetic association studies on human fear learning- and extinction-related processes, as well as their clinical translations, two sets of findings clearly stand out.

First, there is now strong evidence (six positive reports (PR)) that genetic variation in the 5-HTT gene affects conditionability, in the sense of facilitated and possibly more persistent fear conditioning in individuals with putative low 5-HTT expression (5-HTTLPR s-allele or $\mathrm{L}_{\mathrm{G}}$-carriers), and that these individuals are also characterized by vulnerability to PTSD after trauma and possibly more severe clinical symptom profiles.

Second, there is good evidence (4PR, 1 negative report) that genetic variation in the COMT gene affects fear memory consolidation, in the sense of stronger and extinction-resistant fear memories in met-allele carriers, as well as associated increases in the risk for PTSD after trauma as well as resistance to exposure-based treatment in $\mathrm{PD}$ patients.

The work on 5-HTTLPR and COMTval158met draws an impressive line between pharmacological work in vitro, animal models, human molecular genetics, behavioral genetics and clinical studies and support the validity of the moleculargenetic association study approach.

The available literature on the BDNFval66met genotype and conditioning- and extinction-related processes is paved by contradictory and unclear findings, and requires, given high clinical interest and promising animal work, further systematic studies in humans.

Other observations of high potential interest, which however require further confirmation and mechanistic clarification, concern associations of genetic variants in the DAT1 gene (1 PR) in extinction and of the ADCYAP1R1 gene (1PR) in conditioning in females. In addition, there is weak evidence for associations with the DRD2 C957T polymorphism (1PR) and the DRD4 VNTR (1PR), whereas single negative results were reported for the MAO-A VNTR, ANKK1 Taq1A 
restriction fragment length polymorphism and the NSPR1 Asn ${ }^{107}$ lle SNP.

Translation of experimental findings into the clinical context is important and genetic association studies on the outcome of CBT were found for the (triallelic) 5-HTTLPR (1PR in PTSD, 1 negative report in $\mathrm{PD}$ ) and the COMTval158met polymorphism (1PR in PD), and both are also associated with the PTSD development after single or multiple traumata (2PR), whereas experimental exposure has been associated with the NPSR1 Asn ${ }^{107}$ lle polymorphism (1PR).

Studying conditioning: methodological aspects. Where necessary for an informed interpretation of the results, we have addressed choices of outcome measures, data reporting and study design, which, like many other methodological aspects (data preprocessing, data reduction, scoring, statistical analysis), differed considerably between studies (see also Table 3). Methodological variation is inevitable because every study is optimized for the specific question it is supposed to answer. Nevertheless, observance of some critical rules might help increase comparability between studies.

Perhaps most importantly, a formal statistical comparison of outcome scores between groups is an absolute requirement for inferring genotype effects, whereas relying solely on separate analyses for each different group is not informative. Of similar importance is the decision which scores to report. Specifically, in differential conditioning experiments, CS + I CS - contrasts as well as separate reporting of CS + and CS - responses can provide valuable information about excitatory $(\mathrm{CS}+)$ and inhibitory ( $\mathrm{CS}-$ ) mechanisms, as well as general reactivity and sensitization effects. In this context, it is helpful to be aware that different indicators of fear learning tap slightly different processes and involve different neurobiological pathways, which is important for their interpretation. For instance, FPS, in contrast to SCR, is not only sensitive to the arousing properties of a stimulus but also to its valence, in that it is specifically potentiated by unpleasant or aversive stimuli ${ }^{88}$ and inhibited by positive stimuli. ${ }^{89}$ Furthermore, FPS facilitates translation of results from animal to human work given the well-delineated neural pathway involved in startle potentiation and the similar measurements employable in both species. ${ }^{88}$ In this context, positive results in FPS in combination with negative results in SCR in several of the reviewed studies stick out. In addition to physiological indices, self-report measures (fear or shock expectancy ratings) can be informative, in particular as a manipulation check or in case subjective experience is of specific interest. However, their subjective nature renders them inherently vulnerable to experimenter demand and it may thus be important to provide accompanying information about a possible genotype influence on tendencies for reporting in a socially desirable manner (for example, quantified using appropriate questionnaires; ref. 90). In addition, the sharpening of contingency awareness that is induced by such ratings needs to be traded against the gain of information. We would also contend that data reporting should ideally include all experimental phases. For example, when solely interested in extinction, results from the conditioning phase (or any preceding phase) need to be reported in the same measurement modality to rule out preexisting group differences. Finally, we would like to draw the reader's attention to useful guidelines for psychophysiological data recording and analysis (http://www.sprweb.org/journal/ index.cfm\#guidelines).

A more specific issue is whether extinction should be conducted immediately following the conditioning phase or after a delay (for example, $24 \mathrm{~h}$ ). Animal work has suggested that a distinction between immediate and delayed extinction is critical, as only the latter may involve inhibition processes. Immediate extinction may in turn lead to an erasure of the learned responses, ${ }^{91}$ although mixed evidence has emerged lately from human research. ${ }^{92,93}$ Critically, human studies mostly apply immediate extinction, whereas extinction commonly does not occur immediately after conditioning in animal studies or natural contexts, posing problems for translation of findings.

This brief and non-exhaustive discussion of what might appear to be small methodological details, which yet can have strong bearing on results, highlights the need for a detailed and comprehensive reporting of experimental procedures. Tables 2 and 3 have been included in an effort to enable the reader to draw his/her own conclusions, to facilitate comparisons and to provide an initial basis for the planning of future studies.

The association approach: limitations and suggestions. Notwithstanding the apparent successes of the genetic approach to human fear conditioning, some important limitations should be kept in mind when interpreting the results. The strongest limitation lies in the inherently correlative nature of association studies, precluding conclusions about causality. This is a particular concern when chance co-variation of a polymorphism with other potentially causal factors (other genetic variants, personality characteristics) can never be fully excluded or when no heritability measurements are available yet. Enlargement of sample sizes and reproduction in independent cohorts can to some extent protect against such confounds. In this context, it is worth noting that only four studies $27,37,75$ reported negative results (for a particular polymorphism or measure), but all also included positive results for other polymorphisms ${ }^{27,28,62}$ or measures. ${ }^{75}$ Thus, to date, there is no single publication reporting negative findings, raising concerns about publication bias.

The need for replication studies is also highlighted by the fact that genotyping mostly was performed a posteriori (see Table 2). This often resulted in unequal genotype distributions (reflecting population allele frequencies) and multiple testing of identical samples. Therefore, replication studies should ideally be carried out in independent study populations. Generally, a prospective genotyping approach where participants are selected based on genotype and a priori hypotheses, and where genotype groups are matched for potentially relevant characteristics (for example, gender, ethnicity, socioeconomic status, personality measures), can be considered advantageous and provide more statistical power. We would also like to draw the reader's attention to the recommendations of the 'STrengthening the REporting of Genetic Association studies (STREGA) initiative'. 94 
Future directions. So far, most genetic association studies in the field of fear conditioning and extinction have tapped only very basic processes. Future studies should include more fine-grained analyses of learning and extinction processes, for example, by discriminating between extinction learning and extinction recall, ${ }^{95}$ and by disentangling sensitization, consolidation and retention effects from true within-session learning effects. In particular, one major characteristic of learning has so far has been neglected with few exceptions: ${ }^{46}$ change over time within an experimental phase (that is, for instance, changes across the trials of a conditioning phase). Instead, data were presented mostly as the mean of all reactions per experimental phase separately for the CS + and CS-, and the difference between both means. Although this approach is by no means incorrect or uninformative, it limits interpretation of the data by a loss of resolution in time.

In general, the specificity of the findings to fear- and anxietyrelevant processes remains to be addressed. First, neurotransmitters have widespread pleiotrophic effects on biological processes as well as behavior and disease. Thus, the subtle changes in one bottleneck of the system induced by functional polymorphisms in a single gene cannot be expected to be more specific than the systems' general function. Thus, we are not searching for a 'gene for fear learning' or a 'gene for extinction', but rather for modulators on the DNA level.

However, also these modulators (for example, polymorphisms) rarely induce highly specific functional effects and it cannot be neglected that genetic polymorphisms are carried by an individual from the very early stages of embryonic existence, allowing the organism-in contrast to acute pharmacological interventions - to adapt to, and compensate for, small shifts in the functionality of a molecular system, both within and between systems. Hence, group differences associated with a polymorphism in transmitter system A might theoretically also be related to compensatory adaptations in transmitter system $B$. A related concern is that functional effects that are observed in vitro do not necessarily map one-to-one on in vivo functioning, due to possible compensatory mechanisms.

Although studies have so far been relying on the study of single polymorphism candidates or the study of multiple 'unrelated' polymorphisms in the same sample, 'systemic haplotypes' are likely to provide interesting new information and partly overcome this limitation. Combining functional polymorphisms in critical bottlenecks of a single (transmitter) system and a subsequent grouping of individuals based on inferred functional status of the system may be a promising approach for future studies. Studies on single gene or single polymorphism associations may also generate hypotheses for subsequent pharmacological challenge studies, and together, both may provide convergent evidence for the involvement of a molecular pathway. In animals, an interesting alternative approach to association studies are gene expression studies, which give a better picture of the underlying biological pathways and mechanisms than genetics. ${ }^{96}$ Still, it remains an unresolved challenge to identify gene expression patterns associated with learning processes in specific regions of the living human brain. Although human research so far mostly relies on tools like genetic association studies, functional brain imaging and pharmacological challenge tests to unravel the neurobiology of fear learning and extinction, animal work, where gene expression studies are easily feasible, can provide priority candidate genes and blood biomarkers that call to be tested in humans. ${ }^{96}$ In this vein, a recent article by Le-Niculescu and co-workers ${ }^{96}$ provides a list of candidate genes for anxiety disorders identified using a convergent functional genomics approach, whereof very few (for example, DRD2) have been investigated with respect to human fear conditioning and extinction or related clinical phenomena.

In sum, translational work employing a synergy between molecular genetics, neuroimaging, psychophysiology, psychopharmacology and, possibly also, neuroendocrinology will be powerful in unraveling the neurobiology of fear learning and extinction processes. Because a significant proportion of patients do not respond to or tolerate standard treatments, such advances may ultimately open up perspectives for new pharmacological interventions targeted at specific neurobiological pathways or genes as they activate during specific therapeutic learning and memory processes. Hence, combining pharmacological target-specificity with temporal processspecificity in the administration regimen should allow us to increase the efficacy of existing learning-based treatments, as in pharmacological enhancement of CBT (as already seen for D-cycloserine ${ }^{97}$ and cortisol. ${ }^{98}$ Although the study of CBT is still in its infancy, and suffers from the absence of evidence that CBT responsiveness is heritable, it holds big hopes for better anxiety treatments in the future.

\section{Conflict of interest}

The authors declare no conflict of interest.

Acknowledgements. We thank KA Raczka for providing the supplementary data. This work was funded by the Deutsche Forschungsgemeinschaft (Grant DFG KA1623/4-1 (TL, RK); Emmy Noether group DFG KA1623/3-1 (RK))

1. Ohman A, Mineka S. Fears, phobias, and preparedness: toward an evolved module of fear and fear learning. Psychol Rev 2001; 108: 483-522.

2. Mineka S, Zinbarg R. A contemporary learning theory perspective on the etiology of anxiety disorders-It's not what you thought it was. Am Psychol 2006; 61: 10-26.

3. Bouton ME, Mineka S, Barlow DH. A modern learning theory perspective on the etiology of panic disorder. Psychol Rev 2001; 108: 4-32.

4. Lissek S, Powers A, McClure E, Phelps E, Woldehawariat G, Grillon C et al. Classical fear conditioning in the anxiety disorders: a meta-analysis. Behav Res Ther 2005; 43: 1391-1424.

5. Anderson KC, Insel TR. The promise of extinction research for the prevention and treatment of anxiety disorders. Biol Psychiatry 2006; 60: 319-321.

6. Foa EB, Franklin ME, Moser J. Context in the clinic: how well do cognitive-behavioral therapies and medications work in combination? Biol Psychiatry 2002; 52: 987-997.

7. Otto M, Smits J, Reese H. Cognitive-behavioral therapy for the treatment of anxiety disorders. J Clin Psychiatry 2004; 65: 34-41.

8. Linden DEJ. How psychotherapy changes the brain-the contribution of functional neuroimaging. Mol Psychiatry 2006; 11: 528-538.

9. Kandel ER. Biology and the future of psychoanalysis: a new intellectual framework for psychiatry revisited. Am J Psychiatry 1999; 156: 505-524.

10. Hettema JM, Annas P, Neale MC, Kendler KS, Fredrikson M. A twin study of the genetics of fear conditioning. Arch Gen Psychiatry 2003; 60: 702-708.

11. Merrill KA, Steinmetz JE, Viken RJ, Rose RJ. Genetic influences on human conditionability: a twin study of the conditioned eyeblink response. Behav Genet 1999; 29: 95-102.

12. Royce JR. Avoidance conditioning in nine strains of inbred mice using optimal stimulus parameters. Behav Genet 1972; 2: 107-110.

13. Gordon JA, Hen R. Genetic approaches to the study of anxiety. Annu Rev Neurosci 2004 27: 193-222. 
14. Fanselow M, Poulos A. The neuroscience of mammalian associative learning. Annu Rev Psychol 2005; 56: 207-234.

15. Myers K, Davis M. Behavioral and neural analysis of extinction. Neuron 2002; 36 : 567-584.

16. Mechias M-L, Etkin A, Kalisch R. A meta-analysis of instructed fear studies: implications for conscious appraisal of threat. Neurolmage 2010; 49: 1760-1768.

17. Lesch K, Mossner R. Genetically driven variation in serotonin uptake: is there a link to affective spectrum, neurodevelopmental, and neurodegenerative disorders? Biol Psychiatry 1998; 44: 179-192.

18. Berry M, Juorio A, Paterson I. The functional-role of monoamine oxidase-A and oxidase-B in the mammalian central-nervous-system. Progr Neurobiol 1994; 42: 375-391.

19. Heils A, Teufel A, Petri S, Seemann M, Bengel D, Balling U et al. Functional promoter and polyadenylation site mapping of the human serotonin (5-HT) transporter gene. J Neural Transm Gen Sect 1995; 102: 247-254.

20. Parsey R, Hastings R, Oquendo M, Hu X, Goldman D, Huang Y et al. Effect of a triallelic functional polymorphism of the serotonin-transporter-linked promoter region on expression of serotonin transporter in the human brain. Am J Psychiatry 2006; 163: 48-51.

21. Reimold M, Smolka M, Schumann G, Zimmer A, Wrase J, Mann K et al. Midbrain serotonin transporter binding potential measured with [C-11]DASB is affected by serotonin transporter genotype. J Neural Transm 2007; 114: 635-639.

22. Van Dyck C, Malison R, Staley J, Jacobsen L, Seibyl J, Laruelle M et al. Central serotonin transporter availability measured with [l-123]beta-CIT SPECT in relation to serotonin transporter genotype. Am J Psychiatry 2004; 161: 525-531.

23. Nordquist N, Oreland L. Serotonin, genetic variability, behaviour, and psychiatric disorders-a review. Ups J Med Sci 2010; 115: 2-10.

24. Kraft J, Slager S, McGrath $P$, Hamilton S. Sequence analysis of the serotonin transporter and associations with antidepressant response. Biol Psychiatry 2005; 58: 374-381.

25. Wendland J, Martin B, Kruse M, Lesch K, Murphy D. Simultaneous genotyping of four functional loci of human SLC6A4, with a reappraisal of 5-HTTLPR and rs25531. Mol Psychiatry 2006; 11: 224-226.

26. Hu X, Lipsky R, Zhu G, Akhtar L, Taubman J, Greenberg B et al. Serotonin transporter promoter gain-of-function genotypes are linked to obsessive-compulsive disorder. Am J Hum Genet 2006; 78: 815-826.

27. Garpenstrand $\mathrm{H}$, Annas $\mathrm{P}$, Ekblom J, Oreland L, Fredrikson $M$. Human fear conditioning is related to dopaminergic and serotonergic biological markers. Behav Neurosci 2001; 115: 358-364.

28. Lonsdorf T, Weike A, Nikamo P, Schalling M, Hamm A, Ohman A. Genetic gating of human fear learning and extinction: possible implications for gene-environment interaction in anxiety disorder. Psychol Sci 2009; 20: 198-206.

29. Crisan L, Pana S, Vulturar R, Heilman R, Szekely R, Druga B et al. Genetic contributions of the serotonin transporter to social learning of fear and economic decision making. Soc Cogn Affect Neurosci 2009; 4: 399-408.

30. Olsson A, Nearing K, Phelps E. Learning fears by observing others: the neural systems of social fear transmission. Soc Cogn Affect Neurosci 2007; 2: 3-11.

31. Blechert J, Michael T, Vriends N, Margraf J, Wilhelm F. Fear conditioning in posttraumatic stress disorder: evidence for delayed extinction of autonomic, experiential, and behavioura responses. Behav Res Ther 2007; 45: 2019-2033.

32. Kilpatrick D, Koenen K, Ruggiero K, Acierno R, Galea S, Resnick H et al. The serotonin transporter genotype and social support and moderation of posttraumatic stress disorder and depression in hurricane-exposed adults. Am J Psychiatry 2007; 164: 1693-1699.

33. Koenen K, Aiello A, Bakshis E, Amstadter A, Ruggiero K, Acierno R et al. Modification of the association between serotonin transporter genotype and risk of posttraumatic stress disorder in adults by county-level social environment. Am J Epidemiol 2009; 169: 704-711.

34. Kolassa I, Ertl V, Eckart C, Glockner F, Kolassa S, Papassotiropoulos A et al. Association study of trauma load and SLC6A4 promoter polymorphism in posttraumatic stress disorder: evidence from survivors of the Rwandan genocide. J Clin Psychiatry 2010a; 71: 543-547.

35. Bryant RA, Felmingham KL, Falconer EM, Pe Benito L, Dobson-Stone C, Pierce KD et al. Preliminary evidence of the short allele of the serotonin transporter gene predicting poor response to cognitive behavior therapy in posttraumatic stress disorder. Biol Psychiatry 2010; $67:$ 1217-1219

36. Lonsdorf T, Ruck C, Bergstrom J, Andersson G, Ohman A, Schalling M et al. The symptomatic profile of panic disorder is shaped by the 5 -HTTLPR polymorphism. Progr Neuro-Psychopharmacol Biol Psychiatry 2009; 33: 1479-1483.

37. Lonsdorf TB, Rück C, Bergström J, Andersson G, Ohman A, Lindefors $\mathrm{N}$ et al. The COMTval158met polymorphism is associated with symptom relief during exposure-based cognitive-behavioral treatment in panic disorder. BMC Psychiatry 2010a; 10: 99

38. Sabol S, Hu S, Hamer D. A functional polymorphism in the monoamine oxidase A gene promoter. Hum Genet 1998; 103: 273-279.

39. Deckert J, Catalano M, Syagailo Y, Bosi M, Okladnova O, Di Bella D et al. Excess of high activity monoamine oxidase $A$ gene promoter alleles in female patients with panic disorder. Hum Mol Genet 1999; 8: 621-624.

40. Mannisto P, Kaakkola S. Catechol-O-methyltransferase (COMT): biochemistry, molecular biology, pharmacology, and clinical efficacy of the new selective COMT inhibitors. Pharmacol Rev 1999; 51: 593-628.

41. Tenhunen J, Salminen M, Lundström K, Kiviluoto T, Savolainen R, Ulmanen I. Genomic organization of the human catechol O-methyltransferase gene and its expression from two distinct promoters. Eur J Biochem 1994; 223: 1049-1059.

42. Weinshilboum RM, Raymond FA. Inheritance of low erythrocyte catechol-omethyltransferase activity in man. Am J Hum Genet 1977; 29: 125-135.
43. Tunbridge EM, Harrison PJ, Weinberger DR. Catechol-o-methyltransferase, cognition, and psychosis: Val158Met and beyond. Biol Psychiatry 2006; 60: 141-151.

44. Bilder RM, Volavka J, Lachman HM, Grace AA. The catechol-O-methyltransferase polymorphism: relations to the tonic-phasic dopamine hypothesis and neuropsychiatric phenotypes. Neuropsychopharmacology 2004; 29: 1943-1961.

45. Nolan KA, Bilder RM, Lachman HM, Volavka J. Catechol O-methyltransferase Val158Me polymorphism in schizophrenia: differential effects of Val and Met alleles on cognitive stability and flexibility. Am J Psychiatry 2004; 161: 359-361.

46. Raczka KA, Mechias M-L, Gartmann N, Reif A, Deckert J, Pessiglione M et al. Empirica support for an involvement of the meso-striataldopamine system in human fear extinction. Transl Psychiatry 2011; 1: e12; doi:10.1038/tp.2011.10.

47. LaLumiere RT, Nawar EM, McGaugh JL. Modulation of memory consolidation by the basolateral amygdala or nucleus accumbens shell requires concurrent dopamine receptor activation in both brain regions. Learn Mem 2005; 12: 296-301.

48. Kolassa I-T, Kolassa S, Ertl V, Papassotiropoulos A, De Quervain DJ-F. The risk of posttraumatic stress disorder after trauma depends on traumatic load and the catechol-omethyltransferase Val(158)Met polymorphism. Biol Psychiatry 2010b; 67: 304-308.

49. Plomin R, DeFries JC, Loehlin JC. Genotype-environment interaction and correlation in the analysis of human behavior. Psychol Bull 1977; 84: 309-322.

50. Valente NLM, Vallada H, Cordeiro Q, Bressan RA, Andreoli SB, Mari JJ et al. CatecholO-methyltransferase (COMT) val158met polymorphism as a risk factor for PTSD after urban violence. J Mol Neurosci [Internet] 2010; available from: http://www.ncbi.nlm.nih.gov/ pubmed/2108010 (cited 16 February 2011).

51. Cragg SJ, Rice ME. DAncing past the DAT at a DA synapse. Trends Neurosci 2004; 27 270-277.

52. Fuke S, Suo S, Takahashi N, Koike H, Sasagawa N, Ishiura S. The VNTR polymorphism of the human dopamine transporter (DAT1) gene affects gene expression. Pharmacogenomics J 2001; 1: 152-156.

53. VanNess S, Owens M, Kilts $\mathrm{C}$. The variable number of tandem repeats element in DAT1 regulates in vitro dopamine transporter density. BMC Genet 2005; 6: 55.

54. Miller GM, Madras BK. Polymorphisms in the $3^{\prime}$-untranslated region of human and monkey dopamine transporter genes affect reporter gene expression. Mol Psychiatry 2002; 7: 44-55

55. Heinz A, Goldman D, Jones DW, Palmour R, Hommer D, Gorey JG et al. Genotype influences in vivo dopamine transporter availability in human striatum. Neuropsychopharmacology 2000; 22: 133-139.

56. Mill J, Asherson P, Craig I, D'Souza U. Transient expression analysis of allelic variants of a VNTR in the dopamine transporter gene (DAT1). BMC Genet 2005; 6: 3

57. Jacobsen LK, Staley JK, Zoghbi SS, Seibyl JP, Kosten TR, Innis RB et al. Prediction of dopamine transporter binding availability by genotype: a preliminary report. Am J Psychiatry 2000; 157: 1700-1703.

58. Van Dyck C, Malison R, Staley J, Jacobsen L, Seibyl J, Laruelle M et al. Central serotonin transporter availability measured with [l-123]beta-CIT SPECT in relation to serotonin transporter genotype. Am J Psychiatry 2004; 161: 525-531.

59. Schultz W. Behavioral theories and the neurophysiology of reward. Annu Rev Psychol 2006; 57: 87-115.

60. Duan J, Wainwright M, Comeron J, Saitou N, Sanders A, Gelernter J et al. Synonymous mutations in the human dopamine receptor D2 (DRD2) affect mRNA stability and synthesis of the receptor. Hum Mol Genet 2003; 12: 205-216.

61. Hirvonen MM, Laakso A, Någren K, Rinne JO, Pohjalainen T, Hietala J. C957T polymorphism of dopamine D2 receptor gene affects striatal DRD2 in vivo availability by changing the receptor affinity. Synapse 2009; 63: 907-912.

62. Huertas E, Ponce G, Koeneke M, Poch C, Espana-Serrano L, Palomo T et al. The D2 dopamine receptor gene variant C957T affects human fear conditioning and aversive priming. Genes Brain Behav 2010; 9: 103-109.

63. Van Tol HH, Wu CM, Guan HC, Ohara K, Bunzow JR, Civelli $\mathrm{O}$ et al. Multiple dopamine D4 receptor variants in the human population. Nature 1992; 358: 149-152.

64. Asghari V, Sanyal S, Buchwaldt S, Paterson A, Jovanovic V, Van Tol HH. Modulation of intracellular cyclic AMP levels by different human dopamine D4 receptor variants. J Neurochem 1995; 65: 1157-1165.

65. Ding Y-C, Chi H-C, Grady DL, Morishima A, Kidd JR, Kidd KK et al. Evidence of positive selection acting at the human dopamine receptor D4 gene locus. Proc Natl Acad Sci USA 2002; 99: 309-314

66. Wang E, Ding Y-C, Flodman P, Kidd JR, Kidd KK, Grady DL et al. The genetic architecture of selection at the human dopamine receptor D4 (DRD4) gene locus. Am J Hum Genet 2004; 74: 931-944.

67. Poo MM. Neurotrophins as synaptic modulators. Nat Rev Neurosci 2001; 2: 24-32.

68. Chen Z-Y, Patel PD, Sant G, Meng C-X, Teng KK, Hempstead BL et al. Variant brain-derived neurotrophic factor (BDNF) (Met66) alters the intracellular trafficking and activity-dependent secretion of wild-type BDNF in neurosecretory cells and cortical neurons. J Neurosci 2004; 24: 4401-4411.

69. Egan MF, Kojima M, Callicott JH, Goldberg TE, Kolachana BS, Bertolino A et al. The BDNF val66met polymorphism affects activity-dependent secretion of BDNF and human memory and hippocampal function. Cell 2003; 112: 257-269.

70. Lu B, Gottschalk W. Modulation of hippocampal synaptic transmission and plasticity by neurotrophins. Prog Brain Res 2000; 128: 231-241.

71. Rattiner LM, Davis M, Ressler KJ. Brain-derived neurotrophic factor in amygdaladependent learning. Neuroscientist 2005; 11: 323-333. 
72. Hajcak G, Castille C, Olvet D, Dunning J, Roohi J, Hatchwell E. Genetic variation in brain-derived neurotrophic factor and human fear conditioning. Genes Brain Behav 2009 8: 80-85.

73. Lonsdorf T, Weike A, Golkar A, Schalling M, Hamm A, Ohman A. Amygdala-dependent fear conditioning in humans is modulated by the BDNFval66met polymorphism. Behav Neurosci 2010b; 124: 9-15.

74. Soliman F, Glatt CE, Bath KG, Levita L, Jones RM, Pattwell SS et al. A genetic varian BDNF polymorphism alters extinction learning in both mouse and human. Science 2010; 327: 863-866.

75. Raczka K, Gartmann N, Mechias M, Reif A, Buchel C, Deckert J et al. A neuropeptide S receptor variant associated with overinterpretation of fear reactions: a potential neurogenetic basis for catastrophizing. Mol Psychiatry 2010; 15: 1067-1074.

76. Neville MJ, Johnstone EC, Walton RT. Identification and characterization of ANKK1: a novel kinase gene closely linked to DRD2 on chromosome band 11q23.1. Hum Mutat 2004; 23: 540-545.

77. Jonsson E, Nothen M, Grunhage F, Farde L, Nakashima Y, Propping P et al. Polymorphisms in the dopamine D2 receptor gene and their relationships to striatal dopamine receptor density of healthy volunteers. Mol Psychiatry 1999; 4: 290-296.

78. Pohjalainen T, Rinne J, Nagren K, Lehikoinen P, Anttila K, Syvalahti E et al. The A1 allele of the human D-2 dopamine receptor gene predicts low D-2 receptor availability in healthy volunteers. Mol Psychiatry 1998; 3: 256-260.

79. Huertas E, Ponce G, Koeneke M, Poch C, Espana-Serrano L, Palomo T et al. The D2 dopamine receptor gene variant C957T affects human fear conditioning and aversive priming. Genes Brain Behav 2010; 9: 103-109.

80. Reinscheid RK, Xu Y-L, Civelli O. Neuropeptide S: a new player in the modulation of arousal and anxiety. Mol Interv 2005; 5: 42-46.

81. Bernier V, Stocco R, Bogusky M, Joyce J, Parachoniak C, Grenier K et al. Structurefunction relationships in the neuropeptide $S$ receptor-molecular consequences of the asthma-associated mutation N107I. J Biol Chem 2006; 281: 24704-24712.

82. Reinscheid R, Xu Y, Okamura N, Zeng J, Chung S, Pai R et al. Pharmacological characterization of human and murine neuropeptide $S$ receptor variants. J Pharmacol Exp Therap 2005; 315: 1338-1345.

83. Domschke K, Reif A, Weber H, Richter J, Hohoff C, Ohrmann P et al. Neuropeptide S receptor gene-converging evidence for a role in panic disorder. Mol Psychiatry [Internet] 2010; available from: http://dx.doi.org/10.1038/mp.2010.81 (cited 6 January 2011).

84. Beck A, ff EG, Greenberg RLR. Anxiety Disorders, Phobias. Basic Books: New York, 1985

85. Reiss S, McNally R. Theoretical issues in behavior therapy. Reiss S, Bootzin RR (eds) In: The Expectancy Model of Fear. Academic Press: New York, 1985, pp 107-122.

86. Miyata A, Arimura A, Dahl R, Minamino N, Uehara A, Jiang L et al. Isolation of a novel-38 residue-hypothalamic polypeptide which stimulates adenylate-cyclase in pituitary-cells. Biochem Biophys Res Commun 1989; 164: 567-574.
87. Ressler K, Mercer K, Bradley B, Jovanovic T, Mahan A, Kerley K et al. Post-traumatic stress disorder is associated with PACAP and the PAC1 receptor. Nature 2011; 470: 492-497.

88. Hamm AO, Greenwald MK, Bradley MM, Lang PJ. Emotional learning, hedonic change, and the startle probe. J Abnorm Psychol 1993; 102: 453-465.

89. Skolnick Al, Davidson RI. Affective modulation of eyeblink startle with reward and threat. Psychophysiology 2002; 39: 835-850.

90. Crowne DP, Marlowe D. A new scale of social desirability independent of psychopathology. $J$ Consult Psychol 1960; 24: 349-354.

91. Myers KM, Ressler KJ, Davis M. Different mechanisms of fear extinction dependent on length of time since fear acquisition. Learn Mem 2006; 13: 216-223.

92. Huff NC, Hernandez JA, Blanding NQ, LaBar KS. Delayed extinction attenuates conditioned fear renewal and spontaneous recovery in humans. Behav Neurosci 2009; 123: 834-843

93. Norrholm SD, Vervliet B, Jovanovic T, Boshoven W, Myers KM, Davis M et al. Timing of extinction relative to acquisition: a parametric analysis of fear extinction in humans. Behav Neurosci 2008; 122: 1016-1030.

94. Little J, Higgins JPT, loannidis JPA, Moher D, Gagnon F, von Elm E et al. STrengthening the reporting of genetic association studies (STREGA): an extension of the STROBE statement. Ann Intern Med 2009; 150: 206-215.

95. Plendl W, Wotjak $C$. Dissociation of within- and between-session extinction of conditioned fear. J Neurosci 2010; 30: 4990-4998.

96. Le-Niculescu H, Balaraman Y, Patel SD, Ayalew M, Gupta J, Kuczenski R et al. Convergent functional genomics of anxiety disorders: translational identification of genes, biomarkers, pathways and mechanisms. Transl Psychiatry 2011; 1: e9.

97. Grillon C. -cycloserine facilitation of fear extinction and exposure-based therapy might rely on lower-level, automatic mechanisms. Biol Psychiatry 2009; 66: 636-641.

98. de Quervain DJ-F, Bentz D, Michael T, Bolt OC, Wiederhold BK, Margraf J et al. Glucocorticoids enhance extinction-based psychotherapy. Proc Natl Acad Sci [Internet] 2011; available from: http://www.pnas.org/content/early/2011/03/14/1018214108.abstract (cited 5 May 2011)

99. Bechara A, Tranel D, Damasio H, Adolphs R, Rockland C, Damasio AR. Double dissociation of conditioning and declarative knowledge relative to the amygdala and hippocampus in humans. Science 1995; 269: 1115-1118.

Translational Psychiatry is an open-access journal published by Nature Publishing Group. This work is licensed under the Creative Commons Attribution-NoncommercialNo Derivative Works 3.0 Unported License. To view a copy of this license, visit http://creativecommons.org/licenses/by-nc-nd/3.0/

Supplementary Information accompanies the paper on the Translational Psychiatry website (http://www.nature.com/tp) 